\title{
Design and Analysis of Fully Integrated Differential VCOs
}

\author{
M. Prochaska, A. Belski, and W. Mathis \\ University of Hannover, Institute of Electromagnetic Theory and Microwave Technology, Appelstraße 9A, \\ 30167 Hannover, Germany
}

\begin{abstract}
Oscillators play a decisive role for electronic equipment in many fields - like communication, navigation or data processing. Especially oscillators are key building blocks in integrated transceivers for wired and wireless communication systems. In this context the study of fully integrated differential VCOs has received attention. In this paper we present an analytic analysis of the steady state oscillation of integrated differential VCOs which is based on a nonlinear model of the oscillator. The outcomes of this are design formulas for the amplitude as well as the stability of the oscillator which take the nonlinearity of the circuit into account.
\end{abstract}

\section{Introduction}

The demand for ever-higher frequencies and higher levels of integration poses a challenge for the design and implementation of high frequency oscillators. This holds especially in communication area, for example for UMTS receivers, or data acquisition devices like flash ADCs. Since at gigahertz frequencies most off-chip solutions are rendered impractical, on-die solutions are necessary. Recent developments in the field of semiconductor technology lead to the realizability of integrated LC-Tank differential VCOs (Fig. 1) (van den Tang et al., 2003). Due to the easy implementation of the differential operation and relatively good phase noise, differential VCOs play a decisive role for application (Hajimiri, 1999) - for example in high speed PLL circuits. In this paper a bifurcation analysis of integrated LC oscillators is presented. By using symbolic algorithms, which we have implemented by computer algebra, we analyze the stability as well as we calculate an approximate amplitude. By using analytic methods we can provide a functional dependence of the results on circuit parameters. Our analysis leads to design formulas which can directly be used for the implementation of LC-Tank oscillators.

\section{Nonlinear Analysis}

For the analysis and the design of electrical oscillators mostly linear models are used. It is one of the most important

Correspondence to: M. Prochaska

(prochaska@ tet.uni-hannover.de) restrictions of the linearization that the amplitude is not computable (Tietze and Schenk, 2004). On this account for the calculation of an approximate amplitude are several "ad hoc" methods - e.g. harmonic balance - are common practice (Odyniec, 2002). But the difference between the nonlinear oscillatory system and its linearization is more fundamental. The nonlinearity of an oscillator is an integral part of its functionality (Mathis, 2000). So we use a nonlinear approach in order to take the complete behavior of nonlinear components into account.

The mathematical justification for the significance of the nonlinearity is given by the Hartman Grobman Theorem (Guckenheimer and Holmes, 1983): It is well-known that a nonlinear dynamic system is stable, if its linear part has only eigenvalues with negative real parts. If at least one eigenvalue has a positive real part, an oscillatory circuit is unstable. In those cases a system is called hyperbolic. It is an also well-establish condition for a steady state oscillation that a system has a pair of conjugate complex eigenvalues with vanishing real parts. Related criteria were presented by Barkhausen and Nyquist (Tietze and Schenk, 2004; Odyniec, 2002; Parzen, 1983). In this case a circuit model is called a non-hyperbolic system. The Hartman Grobman Theorem tells us that, if a system is non hyperbolic, we cannot neglect the nonlinearity. The neglect of the nolinearity leads to a loss of accuracy of the underlying circuit model. Thus the necessity of a nonlinear analysis of oscillators is originated in the Hartman Grobman Theorem. On this account we present a nonlinear design methodology for integrated LC-Tank oscillators.

So it is suitable to model electrical oscillators by using a nonlinear dynamic system

$\dot{\mathbf{x}}=\mathbf{f}(\mathbf{x})$,

where $\mathbf{f}: \mathbb{R}^{n} \rightarrow \mathbb{R}^{n}$ and $\mathbf{x} \in \mathbb{R}^{n}$. The vector $\boldsymbol{x}$ corresponds to time depending currents or voltages of the circuit while $f$ is a nonlinear vector field containing the influence of the gain element.

\section{Andronov Hopf Theorem}

In order to apply the Andronov Hopf Theorem it is useful to get an overview of the possible solutions. A planar nonlinear 


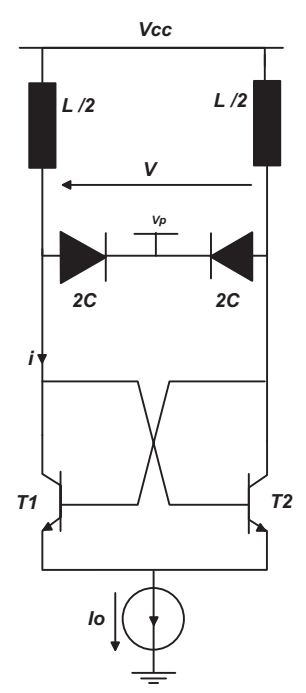

Fig. 1. Fully integrated LC-Tank VCO.

dynamical system has primarily two different types of solutions: equilibrium points and periodic solutions. Equilibrium points are the roots of the nonlinear field $\mathbf{f}(\mathbf{x})$ while periodic solutions are close trajectories in the state space. If a periodic solution is isolated, i.e. isolated in the sense that there are no other close trajectories in its neighborhood, this curve will be called a limit cycle. The relationship between an equilibrium point and a limit cycle is given by the Andronov Hopf Theorem. Originally this theorem was proved by Andronov in 1935 for the analysis of tube oscillators (Mathis, 1998). In this context we consider a dynamic system which additionally depends on the parameter $\mu$ :

$\dot{\mathbf{x}}=\mathbf{f}(\mathbf{x}, \mu)$

For example, $\mu$ represents a component of the circuit like a load resistor. Comprising, the Andronov Hopf Bifurcation describes the birth of a limit cycle depending on $\mu$ in the neighborhood of zero and under the following conditions:

$-\left.\frac{\partial \mathbf{f}}{\partial \mathbf{x}}\right|_{\substack{\overline{\mathbf{x}}=0, \mu=0}}$ has a pair of conjugate complex eigenvalues

- all other eigenvalues possess negative real parts

- $\left.\frac{d}{d \varepsilon} \mathfrak{R} \lambda_{1}(\mu)\right|_{\mu=0}>0$

- the equilibrium point is asymptotic stable

If these conditions are satisfied, there is a stable equilibrium for $\left(-\mu_{1}, 0\right)$ and a stable limit cycle for $\left(0, \mu_{2}\right)$. Thus, the Andronov-Hopf theorem is the basis for the operating mode of oscillators (Mees and Chua, 1979).

\section{Circuit Model}

For a nonlinear analysis of LC-Tank oscillators modeling of the tuning diodes, the bipolar transistors and the monolitic

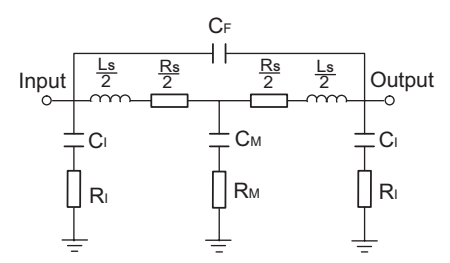

Fig. 2. Model of an Octo-Coil.

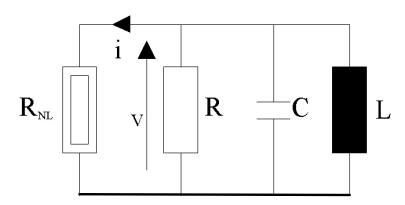

Fig. 3. Fully integrated LC-Tank VCO.

inductors is necessary. In order to get an adequate approximation of our model we shape the transistors by the EbersMoll model. We assume that both transistors are the same and that their parameters are sufficiently known. Furthermore, we neglect the nonlinearity of tuning diodes which we approximate as ideal capacitors.

The on-chip inductors perform a critical role in integrated RF circuits, since their Quality factors are much lower than that of off-chip components. Since the model of integrated inductors depends primarily on the IC process and the implementation, it is not easy to specify a generally applicable model. Moreover, a suitable inductor model leads to heavy calculations of high-dimensional dynamical systems. For example, an adequate equivalent inductor model of an OctoCoil, which can be used for the implementation of a fully integrated LC tuned VCO using a $0.12 \mu \mathrm{m}$ CMOS process, consist of several dynamic components in a double- $\pi$-circuit (Fig. 2) (Konstanznig et al.,2002). That is why we represent the on-chip inductors by ideal electrical devices.

Strategies for the modelization of integrated inductors are given in Arcioni et al. (1999), Brunch et al. (2002) and Danesh and Long (2002). In Prochaska and Mathis (2004) we have presented some ideas for the reduced order modeling of high dimensional oscillators. Figure 3 shows a useful nonlinear model for differential VCOs (Buonomo and Schiavo, 2003).

In order to gain such equivalent circuit of cross-coupled LC-Tank oscillators, first we calculate the bias-emitter voltage of the transistors. After that, we are able to find an equation for the current

$$
\begin{aligned}
& i(v)= \\
& \frac{\alpha_{F} I_{0}}{1+e_{T}^{v / V}}\left(1+\frac{1}{\beta_{F}} e^{v / V_{T}}\right)+\frac{I_{S}}{\alpha_{R}}\left(e^{v / V_{T}}-e^{-v / V_{T}}\right)+\frac{I_{S}}{1+e^{v / V_{T}}} \\
& {\left[\alpha_{F}\left(e^{-v / V_{T}}-e^{2 v / V_{T}}\right)+\left(4-3 \alpha_{F}-\frac{1}{\alpha_{F}}\right)\left(1-e^{v / V_{T}}\right)\right],}
\end{aligned}
$$

which enables us to define a simple equivalent circuit. $\mathrm{R}_{N L}$ 
represents the nonlinearity of the bipolar transistors. So we can calculate the model of the oscillator

$$
\left(\begin{array}{c}
\frac{d v}{d t} \\
\frac{d i_{L}}{d t}
\end{array}\right)=\left(\begin{array}{cc}
-\frac{1}{R C} & -\frac{1}{C} \\
\frac{1}{L} & 0
\end{array}\right)\left(\begin{array}{c}
v \\
i_{L}
\end{array}\right)+\left(\begin{array}{c}
\frac{1}{C} \\
0
\end{array}\right) i(v) .
$$

The model also shows that the popular maximally loaded condition for the design of oscillatory circuits fails (Mathis and Weghorst, 1995). In fact, Odyniec (2002) desribes the general oscillator - i.e. the case of a non-maximum loading.

\section{Analysis of the Stability}

The Andronov Hopf Theorem tells us, that the linear part of an oscillatory system, which is given by the Jacobian $D_{x} \mathbf{f}(\mathbf{0})$ evaluated at the equilibrium point, has to have a pair of conjugate complex eigenvalues with vanishing real parts. The equilibrium point of Eq. (4) is given by $i=i\left(I_{0}\right)$ and $v=0$. In order to guarantee the existence of a pair of pure imaginary eigenvalues, we have to calculate a multidimensional power series of the nonlinear vector field of Eq. (4). We get

$$
\left(\begin{array}{c}
\frac{d v}{d t} \\
\frac{d i_{L}}{d t}
\end{array}\right)=\left(\begin{array}{cc}
-\frac{1}{R C}+\frac{i^{\prime}\left(I_{0}\right)}{C} & -\frac{1}{C} \\
\frac{1}{L} & 0
\end{array}\right)\left(\begin{array}{c}
v \\
i_{L}
\end{array}\right)+\left(\begin{array}{c}
\frac{1}{C} i\left(I_{0}\right) \\
0
\end{array}\right),
$$

where only the linear part of the expansion is treated. If the condition

$$
\frac{i^{\prime}\left(I_{0}\right)}{C}-\frac{1}{R C}=0
$$

holds, Eq. (5) has eigenvalues

$\lambda_{1,2}= \pm j \omega= \pm j \frac{1}{\sqrt{L C}}$.

The first condition given by the Andronov Hopf Theorem is fulfilled which is equivalent to the Barkhausen condition. In this case we find

$$
I=I_{0}=-\frac{2\left(\left(2 \alpha_{R} V_{T}-4 I_{s} R+4 I_{s} R \alpha_{R}\right) \alpha_{F}-I_{s} R \alpha_{R}\right)}{\alpha_{R} \alpha_{F}\left(-1+2 \alpha_{F}\right) R} .
$$

If we choose $I_{0}=I$ the equilibrium point of Eq. (5) is in the origin and so a necessary condition for the applicability of the Andronov Hopf Theorem is also satisfied. The next point, we have to proof that the equilibrium point is asymptotic stable. For the further calculation it is useful to simplify the linear and nonlinear part of our given system. The initial point for the simplification is Eq. (5). The idea is to choose a coordinate transformation so as to simplify the terms of the vector field. In order to simplify the linear part of $\mathbf{f}$, we diagonalize the linear part by

$\dot{\mathbf{y}}=\mathbf{J y}+\mathbf{T}^{-1} \tilde{\mathbf{f}}(\mathbf{T y})$,

with the diagonal matrix $\mathbf{J}=\mathbf{T}^{-1} \mathbf{A T}, \mathbf{x}=\mathbf{T y}$ and $\tilde{\mathbf{f}}$ the nonlinear part of $\mathbf{f}$. The initial point of the analysis of stability of our oscillator is Eq. (5), which can be rewritten as

$$
\left(\begin{array}{l}
\frac{d x_{1}}{d t} \\
\frac{d x_{2}}{d t}
\end{array}\right)=\left(\begin{array}{cc}
\mu\left(I_{0}\right) & -\frac{1}{C} \\
\frac{1}{L} & 0
\end{array}\right)\left(\begin{array}{l}
x_{1} \\
x_{2}
\end{array}\right)+\left(\begin{array}{c}
\frac{1}{C} \gamma x_{1}^{3} \\
0
\end{array}\right),
$$

where $\mathbf{x}=\left[v i_{L}\right]^{T}$ and the bifurcation parameter $\mu$ is given by

$\mu\left(I_{0}\right)=\frac{i^{\prime}\left(I_{0}\right)}{C}-\frac{1}{R C}$

and the constant

$$
\begin{aligned}
& \gamma\left(I_{0}\right)=-\frac{1}{48} \frac{I_{0}}{V_{T}^{3}}+\frac{1}{24} \frac{I_{0}}{V_{T}^{3}} \alpha_{F}+\frac{1}{3} \frac{I_{S}}{\alpha_{R} V_{T}^{3}} \\
& -\frac{1}{2} \frac{I_{S}}{V_{T}^{3}} \alpha_{F}+\frac{1}{6} \frac{I_{S}}{V_{T}^{3}}-\frac{1}{24} \frac{I_{S}}{\alpha_{F} V_{T}^{3}}
\end{aligned}
$$

With $\mathbf{T}=\left[01 ;-1 / \mathrm{C} / \omega_{0} 0\right]$ we find:

$\dot{\mathbf{y}}=\left(\begin{array}{cc}0 & j \omega_{0} \\ -j \omega_{0} & 0\end{array}\right) \mathbf{y}+\left(\begin{array}{c}0 \\ -\frac{1}{C} \gamma y_{1}^{3}\end{array}\right)$.

After that, in order to simplify the nonlinear terms we try to find a sequence of coordinate transformations which remove terms of increasing degree from the Taylor series (Mathis, 1995). So a dynamic system which has eigenvalues $\alpha(\mu) \pm \mathrm{j} \omega(\mu)$ can be expressed in the so called Poincare normal form

$\dot{\mathbf{y}}=\left[\begin{array}{cc}\alpha(\mu) & \omega(\mu) \\ -\omega(\mu) & \alpha(\mu)\end{array}\right] \mathbf{y}+\sum_{i=1}^{\infty}\left(y_{1}^{2}+y_{2}^{2}\right)^{i}\left[\begin{array}{cc}a_{i} & b_{i} \\ -b_{i} & a_{i}\end{array}\right]\left[\begin{array}{l}y_{1} \\ y_{2}\end{array}\right]$

The reader should note that the equilibrium point is asymptotical stable, if the so-called Poincare coefficient $a_{1}$ is negative $\left(a_{1}<0\right)$. The calculation of the Poincare normal form of Eq. (13) for $\mu=0$ gives:

$\dot{\mathbf{y}}=\left[\begin{array}{cc}0 & \omega \\ -\omega & 0\end{array}\right] \mathbf{y}+\left(y_{1}^{2}+y_{2}^{2}\right)\left[\begin{array}{cc}-\frac{3}{8} \gamma & 0 \\ 0 & -\frac{3}{8} \gamma\end{array}\right]\left[\begin{array}{l}y_{1} \\ y_{2}\end{array}\right]+O\left(\|\mathbf{y}\|^{5}\right)$

We get the Poincare coefficient $a_{1}=-3 / 8 \gamma$. For $\gamma>0$ the equilibrium is asymptotic stable. So we can guarantee the asymptotic stability of the equilibrium point. In order to demonstrate the birth of a limit cycle vividly, we analyze the product of the voltage and current of the nonlinear resistor $\mathrm{R}_{N} L i v=f\left(v, I_{0}\right)$ which is shown in Figs. 4 and 5. If we choose $I_{0}=I$, the term $i v$ has locally a negative slope. When $i v$ is interpreted as power the negative slope indicates a negative differential resistor in the neighborhood of the zero solution. This leads to an oscillation since the loss of the circuit will be compensated. It must be pointed out that Eq. (8) also satisfies the condition $d\left(\left.\Re \lambda_{1}(\mu)\right|_{\mu=0}\right) / d \varepsilon>0$ given by the Andronov Hopf Theorem, which can be tested by an easy computation.

\section{Predicting of the Amplitude}

In order to calculate the amplitude of a sinusoidal oscillator it is suitable to transform the reduced system to polar coordinates. Starting from Eq. (9) we obtain the following system for $\mu=0(\mathbf{y} \Leftrightarrow(r, \Theta))$ :

$\left[\begin{array}{c}\dot{\Theta} \\ \dot{r}\end{array}\right]=\left[\begin{array}{c}\omega \\ 0\end{array}\right]+\mathbf{f}_{P C}(\Theta, r)$. 


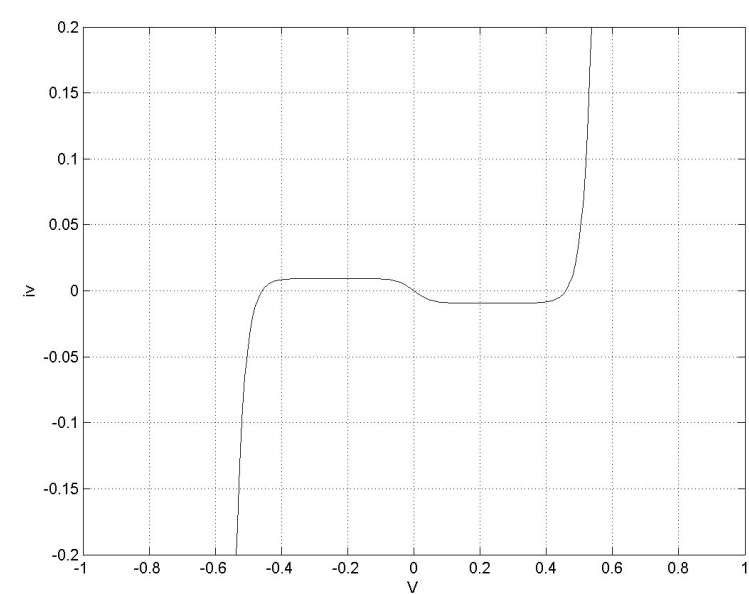

Fig. 4. Analysis of the power $i v$ for $I_{0}=I$.

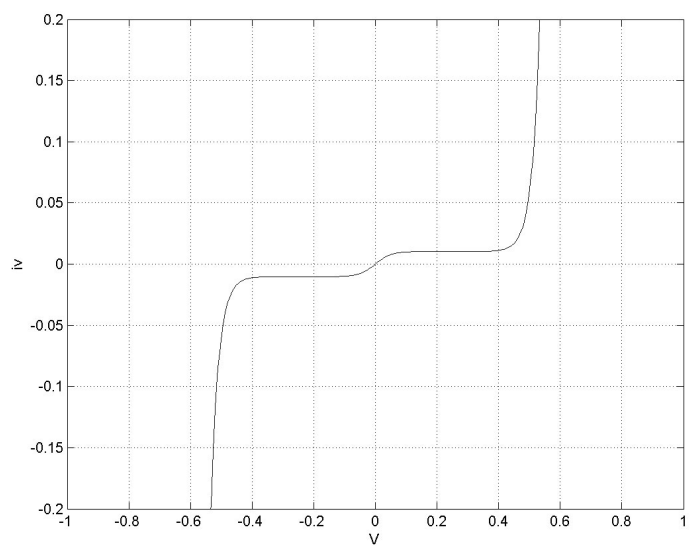

Fig. 5. The power $i v$ for $I_{0}=I$.

Since the equation $\dot{r}=0$ is mostly a function of $\Theta$, i.e. both equations of Eq. (16) are coupled; we cannot calculate the amplitude directly. To produce a relief we use an average technique - a perturbation method (Guckenheimer and Holmes, 1983). Through this method the trajectory of the limit cycle of one period is averaged. Instead of popular "ad hoc" methods averaging is an analytic method; the error of the approximation can be calculated. By means of this technique it is our goal to eliminate the action of $\Theta$ in the second equation of Eq. (16). The method hinges on the identification of a small parameter $\varepsilon$ which marks the perturbation. We assume that a LC circuit is perturbed by a small nonlinearity. So we get in Cartesian coordinates for a planar system with $\mu=0$

$\dot{\mathbf{y}}=\left[\begin{array}{cc}0 & \omega \\ -\omega & 0\end{array}\right] \mathbf{y}+\varepsilon \mathbf{f}_{S}(\mathbf{y})=\mathbf{J} \mathbf{y}+\varepsilon \mathbf{f}_{S}(\mathbf{y})$.

In polar coordinates we can write the following system, where mostly both equations are coupled

$\left[\begin{array}{c}\dot{\Theta} \\ \dot{r}\end{array}\right]=\left[\begin{array}{c}\omega \\ 0\end{array}\right]+\varepsilon\left[\begin{array}{l}R(\Theta, r) \\ T(\Theta, r)\end{array}\right]$.

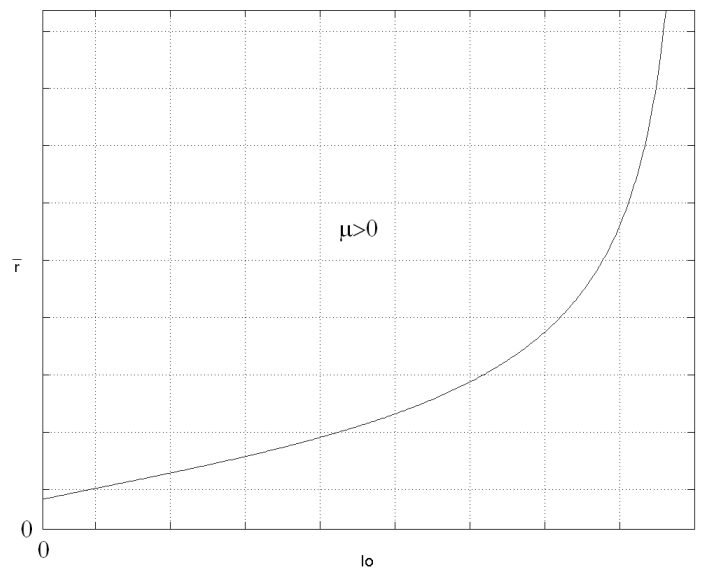

Fig. 6. Qualitative behavior of the amplitude $\bar{r}_{1}=f\left(I_{0}\right)$ in the neighborhood of the bifurcation point for $\mu>0$.

By using a suitable transformation we find the averaged system

$\left[\begin{array}{c}\dot{\bar{\Theta}} \\ \dot{\bar{r}}\end{array}\right]=\left[\begin{array}{c}\omega \\ 0\end{array}\right]+\varepsilon\left[\begin{array}{l}\bar{R}(\bar{r}) \\ \bar{T}(\bar{r})\end{array}\right]$.

Here we have assumed that already one averaging gives a usable result. But sometimes a sequence of coordinate transformations is necessary. Then our approach, which is based upon Lie series, is advantageous (Mathis, 1987). So we can transform Eq. (13) to polar coordinates:

$\left[\begin{array}{c}\dot{\Theta} \\ \dot{r}\end{array}\right]=\left[\begin{array}{c}\omega_{0} \\ 0\end{array}\right]+\varepsilon\left[\begin{array}{c}r^{2} \sin \Theta \cos \Theta \\ r \mu+\mu \cos ^{2} \Theta+\ldots\end{array}\right]$

Since both equations of Eq. (20) are coupled averaging is necessary. We calculate

$\left[\begin{array}{c}\dot{\bar{\Theta}} \\ \dot{\bar{r}}\end{array}\right]=\left[\begin{array}{c}1 \\ \varepsilon \bar{r} \mu-\varepsilon \frac{3}{8} \gamma \bar{r}^{3}\end{array}\right]$,

where already one averaging removes the terms depending on $\Theta$. The roots for $\dot{\bar{r}}=0$ are

$\bar{r}_{1}=0, \bar{r}_{2}= \pm \sqrt{\frac{8}{3} \frac{\mu\left(I_{0}\right)}{\gamma\left(I_{0}\right)}}$.

The value $\bar{r}_{1}=0$ defines the averaged amplitude for an unstable equilibrium. If the Andronov Hopf Theorem is fulfilled, the oscillator has the amplitude $\left|\bar{r}_{2}\right|$, which depends on $\mu\left(I_{0}\right)$ and the parameters of the transistors. Thus, by means of current $I_{0}$ the amplitude can be tuned in the neighborhood of the bifurcation point (Fig. 6). Furthermore, Eq. (22) shows the dependence of the amplitude on the nonlinearity of the transistor.

\section{Conclusion}

In this work we have presented a complete bifurcation analysis of integrated differential LC oscillators. We have shown 
the analysis of the stability of the circuit as well as the calculation of an approximate amplitude. We have presented the results in an analytic form - designers are able to implement differential VCO by means of the given results even if other models of the components of the circuit are required. In this case possibly the calculations are more complicated. However, our methodology can be used in the same way. So it represents a guide for the analysis and the design of electrical oscillators whereas the nonlinearities of circuit components are an integral part of the design process.

It turns also out that geometric methods are powerful tools for investigations of nonlinear oscillators. Since they provide a survey of the solution set we get a deeper insight in the behavior of the network. In contrast to numerical computation the shown analytical methods have the advantage that the results are interpretable by the parameters of the network. Furthermore, we have implemented the geometric methods by means of computer algebra. Because of these routines the calculations proceed in an automated way.

\section{References}

Arcioni P., Castello, R., Perregrine, L., Sacchi, E., and Svelto, F.: An innovative modelization of loss mechanism in silicon integrated inductors, IEEE Trans. On Cir. And Sys., Vol. 46, No. 12, 1453-1460, Dec. 1999.

Brunch R. L., Sanderson, D. I., and Ramon, S.: Quality factor and inductance in differential IC implementations, IEEE Mircrowave Mag., Vol. 3, No. 2, 82-92, June 2002.

Buonomo A. and Schiavo, A. Lo: Determining the oscillation of differential VCOS, Proc. of the 2003 Intern. Sym. on Cir. and Sys. 2003 (ISCAS2003), Vol. 3, 25-28, May 2003.

Danesh, M. and Long, J. R.: Differential driven symmetric microstrip inductors, IEEE Trans. On Microwave Theo. And Tech., Vol. 50, No. 1, 332-341, Jan. 2002.

Guckenheimer, J. and Holmes, P.: Dynamical Systems and Bifurkation of Vector Fields, Springer Verlag New York, 1983.
Hajimiri, A and Lee, T. H.: Design Issues in CMOS Differential LC Oscillators, IEEE Journal. of Solid State Circuits, Vol. 34, No 5, May 1999.

Konstanznig, G., Pappenreiter, T., Maurer, L., Springer, A., and Weigel, R.: Design of a $1.5 \mathrm{~V}, 1.1 \mathrm{~mA}$ Fully Integrated LCtuned Voltage Controlled Oscillator in a $4 \mathrm{Ghz}-$ Band using a $0.12 \mu \mathrm{m}$ CMOS-Process, Asian-Pacific Microwave Conference, 1471-1474, Nov. 2002.

Mathis, W.: Transformation and Equivalence, in: The Circuits and Filters Handbook, edited by Chen, W. K., CRC Press Boca Raton, 1995.

Mathis, W.: Historical Remarks of the History of Electrical Oscillators, Proc. MTNS-98 Symposium, July 1998, 309-312, Padova 1998.

Mathis, W.: Nonlinear electronic circuits - An overview, Proc. 7th., Gdynia, Poland, MIXDES 2000.

Mathis, W. and Weghorst, I.: A Nonlinear Theory for MaximumLoaded-Oscillators with the Andronov Hopf Bifurcation Theorem, SIAM Fall Meeting, 28-30 Oct. 1985, Arizona State University, 1985.

Mathis, W. and Voigt, I. : Applications of Lie Series Averaging in Nonlinear Oscillations, Proc. IEEE Intern. Symp. Of Cir. And Sys. (ISCAS1987), Philadelphia, 1987.

Mees, A. I. and Chua, L. O.: The Hopf Bifurcation and its Application to nonlinear Oscillations in Circuits and Systems, IEEE Trans. Circuits and Systems, vol. 26, 235-254, 1979.

Odyniec, M.: RF and Microwave Oscillator Design, Artech House, Boston, 2002.

Parzen, B.: Design of Crystal and Other Harmonic Osillators, Wiley \& Sons, 1983.

Prochaska M. and Mathis, W.: On limit cycles in singularly perturbed electrical circuits, Proc. of the 16th Int. Sym. on Math. Theory of Netw. and Sys. (MTNS), Leuven 2004.

van der Tang, J., Kasperkovitz, D., and van Roermund, A.: HighFrequency Oscillator Design for Integrated Transceivers, Kluwer Academic Publishers, Boston, 2003.

Tietze, U. and Schenk, C.: Electronic Circuits: Handbook for Design and Application, Springer Verlag, New York, 2004. 